\title{
Relative figure of merit of optical interferometry and spectroscopy
}

\section{Example of parameter estimates of P Cygni circumstellar envelope}

\author{
M.S. Burgin ${ }^{1,2}$ and A. Chalabaev ${ }^{2}$ \\ 1 Astro-Space Center FIAN, Profsoyuznaya 84/32, 117810 Moscow, Russia \\ e-mail: mburgin@dpc.asc.rssi.ru \\ 2 CNRS, Laboratoire d'Astrophysique, Observatoire de Grenoble, UMR 5571, BP. 53X, F-38041 Grenoble Cedex, France \\ e-mail: Almas.Chalabaev@obs.ujf-grenoble.fr
}

Received June 14; accepted September 21, 1999

\begin{abstract}
When planning new facilities, one is interested to know whether and by how much the new technique is superior to already existing ones. We describe a general approach permitting us to evaluate the relative merits of various techniques used in astrophysical observations, following the theory of model parameter estimation. It is applied to compare two-aperture optical long baseline interferometry to classical spectroscopy, both used to define the model parameters of the P Cyg wind. The wind modeling was done using an efficient approximation for computation of the line source function; it allowed us to analyse about $10^{5}$ points in the parametric space of $\mathrm{P}$ Cyg envelope models. It is shown that interferometry offers no real advantage if the object can be described by stationary spherically symmetric models with a priori fixed thermal structure. However, if the object must be described by a model with a large number of free parameters, e.g. when the thermal structure of the envelope is not fixed a priori, then the interferometric measurements can reduce the error in model parameters determination by an order of magnitude. In the case of $\mathrm{P} \mathrm{Cyg}$, the reduction of errors provided by interferometry is highest for the baseline lengths in the range $45-90 \mathrm{~m}$. This illustrates the capacity of the proposed method to be used for optimization of interferometric configurations. The simplifications adopted for this first trial application are critically analyzed, and future improvements are indicated.
\end{abstract}

Key words: techniques: interferometric - techniques: spectroscopic - P Cyg — stars: mass-loss

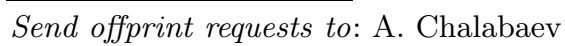

\section{Introduction}

When evaluating justification for new facilities, one may question whether and by how much the new technique is superior to already existing ones. We describe a general approach which leads to a quantitative answer, expressed as a relative figure of merit of two observational techniques. The approach is based on the comparison of the corresponding errors in the model parameters determination. The derived figure of merit can also be used in the closely related problem of optimization of complex observations, thus contributing to maximizing their scientific output.

We illustrate the developed method by comparing the technique of optical long baseline interferometry to the classical spectroscopy, both used to find the parameters of the gaseous envelope around the P Cyg star. For this first trial application, we use simulated data, obtained by solving the radiative transfer problem for grids of physical models of the envelope and then adding the photon noise.

The chosen example of the interferometry vs. spectroscopy comparison appears to be timely. Indeed, in recent years, interferometry has been undergoing steady progress witnessed by publication of fringe visibilities for circumstellar envelopes of various types sometimes combined with a good spectral resolution (Mourard et al. 1989; Vakili et al. 1994, 1997; Quirrenbach et al. 1993, 1994, 1997; Harmanec et al. 1996; Stee et al. 1995; see also expected performances of interferometric arrays under construction, VLTI, von der Lühe et al. 1997; Petrov et al. 1998, and CHARA, McAlister et al. 1997). While these studies are fully justified, for the knowledge of the flux distribution on small angular scales can unveil important new features of astrophysical objects, it is also important to question whether this effort is justified for all classes of objects and for all kinds of physical problems. As a matter of fact, interferometric observations as compared 
to those at a single telescope are more complex, more expensive, more time consuming, and, when possible to compare, have a larger accumulated measurement error due to their complexity. Furthermore, in the foreseeable future the instruments of this type will remain orders of magnitude less numerous and by all given reasons much less available for the community than single telescopes. Thus, it makes sense to evaluate in quantitative terms what are the targets for which the interferometric instrumentation has the highest potential in terms of astrophysical information as compared to single telescopes.

Also, the optimization of interferometric observations may be a crucial aspect. Indeed, the existing and forthcoming long baseline interferometers comprise only few individual apertures, covering a small set of spatial frequencies during one observing cycle. On the other hand, the information provided by observations can depend critically on spatial frequencies. Certainly, in such a situation the scientific yield of the interferometric array could be greatly improved if thorough model calculations can indicate the configurations expected to be the most useful for determination of intended physical parameters of the object.

Not surprisingly, the relative figure of merit derived in the present article depends not only on the experimental techniques, but also on the chosen set of model parameters, reflecting the fact that the physical model makes a necessary part of the merit problem. However, in modern astronomy, designing and building instrumentation on the one hand, and modeling objects on the other hand, were pushed to such a level of sophistication that they are as a rule two distinct activities. The optimum use of interferometry needs a close collaboration of these two communities.

After these preliminary remarks, let us specify that in the present first trial application, our discussion will be limited to the comparison of observations with a twoaperture optical long baseline interferometer (hereafter OLBI) and the classical single telescope spectroscopy (hereafter spectroscopy), both applied to study a circumstellar gaseous envelope formed by the wind of a massive star. The OLBI observations are assumed to have the same spectral resolution as spectroscopy. The discussion will be further limited to spectral profiles and visibilities in the $\mathrm{H} \alpha$ line of the $\mathrm{P}$ Cyg envelope, allowing us to use published high quality observations both in OLBI and spectroscopy, and thus to provide a clear numericnalial illustration.

In our earlier attempts to solve the OLBI vs. spectroscopy merits problem (Burgin \& Chalabaev 1992; Bourguine \& Chalabaev 1994), we used qualitative comparison of observables, visibility and spectral profile, computed for a small number of envelope models. The results were ambiguous. Analysing them, we arrived at the firm conviction that meaningful conclusions can be obtained only if (1) intercomparisons of different types of observations are performed using a clearly defined figure of merit, indicating how much information on the studied object is provided by various techniques, and (2) sufficiently large ranges in the space of envelope model parameters are analyzed.

In the case of $\mathrm{P} \mathrm{Cyg}$, the required number of computed models (see Sect. 5) turned out to be $10^{4}-10^{5}$. An efficient simplified envelope model code, computing the emergent spectral profile and visibilities for the $\mathrm{H} \alpha$ line, was developed and is described in Sect. 4. We considered only spherically symmetric outflow models, the choice of which is justified in Sect. 4.

\section{Relative figure of merit of observational methods}

\subsection{Definitions and general theory}

We consider astrophysical observations and their interpretation in the framework of the theory of parameter estimation (e.g. Kendall \& Stuart 1967). That is, the observed object is assumed to be exactly described by a fixed multiparametric physical model, the errors being solely those of the experimental measurements (see however Sect. 2.3). Any observable quantity is then a function of the vector of $M_{\mathrm{P}}$ model parameters $\Theta=\left(\Theta_{1}, \Theta_{2}, \ldots, \Theta_{M_{\mathrm{P}}}\right)$.

An observation consists of finding the values of $M_{\mathrm{O}}$ observables $\hat{\boldsymbol{Y}}=\left(\hat{Y}_{1}, \hat{Y}_{2}, \ldots, \hat{Y}_{M_{\mathrm{O}}}\right)$, where each observable $\hat{Y}_{i}$ is either measured directly or can be calculated in a modelindependent way as a known function of directly measurable quantities. Obviously, observations with instruments of different types provide observables of different numbers and natures.

The $i$-th observable can be represented as $\hat{Y}_{i}=Y_{i}(\boldsymbol{\Theta})+$ $\varepsilon_{i}$, where $Y_{i}(\boldsymbol{\Theta})$ is the "theoretical" value of the corresponding observable in the absence of observational errors and $\varepsilon=\left(\varepsilon_{1}, \varepsilon_{2}, \ldots, \varepsilon_{M_{\mathrm{O}}}\right)$ is the random vector of observational errors, which is assumed here to be distributed normally. We consider the case when $M_{\mathrm{O}}>M_{\mathrm{P}}$ and the parameters $\Theta$ could have been uniquely determined if the vector $\boldsymbol{Y}$ were known.

We shall call the parameters to be determined the "target parameters" and denote a set of $M_{\mathrm{T}}$ target parameters, or target set, as $\mathcal{T}$. It is possible that $M_{\mathrm{T}}<M_{\mathrm{P}}$ and the set $\mathcal{T}$ is a proper subset of the set of all parameters of the model. A situation of this kind may arise for two reasons.

First, a model parameter may be set to some a priori fixed value because, say, it has been measured earlier by entirely different methods. This reduces the dimension of the problem and computational difficulties, the general method remaining the same. Let $\mathrm{F}$ denote the set of $M_{\mathrm{F}}$ fixed parameters, and $\tilde{\Theta}_{\mathrm{f}}$ be the value a priori assigned to a parameter $\Theta_{\mathrm{f}} \in \mathcal{F}$.

Secondly, some of the unknown physical parameters may eventually be considered "not interesting" if their values are not relevant to the astrophysical problem under study. Following the theory of parameter estimation, 
we will call them the "nuisance" parameters and denote the set of nuisance parameters, or "nuisance set", as $\mathcal{N}$, their number being $M_{\mathrm{N}}$. As a rule, their influence can be separated in the error analysis only at the final stage of computations, for their values are to be calculated along with the values of target parameters.

Interpretation of an observation consists of calculating the vector $\hat{\boldsymbol{\Theta}}_{\mathrm{T}}(\hat{\boldsymbol{Y}})=\left(\hat{\Theta}_{t(1)}, \hat{\Theta}_{t(2)}, \ldots, \hat{\Theta}_{t\left(M_{\mathrm{T}}\right)}\right)$ that provides the best fit to the observed values $\hat{\boldsymbol{Y}}$. Here $t(i)$ is the index of the component of vector $\Theta$ corresponding to the same model parameter as the $i$-th target parameter $\left(i=1, \ldots, M_{\mathrm{T}}\right)$.

The precision of the values $\hat{\boldsymbol{\Theta}}_{\mathrm{T}}$ resulting from interpretation of the observations is characterized by $\mathrm{M}\left(\hat{\boldsymbol{\Theta}}_{\mathrm{T}}-\boldsymbol{\Theta}_{\mathrm{T}}\right)$ and $\mathrm{D} \hat{\boldsymbol{\Theta}}_{\mathrm{T}}$, where for any random vector $\boldsymbol{\xi}$ expressions $\mathrm{M} \boldsymbol{\xi}$ and $D \boldsymbol{\xi}$ denote its mean and its covariance matrix respectively, and $\boldsymbol{\Theta}_{\mathrm{T}}=\left(\Theta_{t(1)}, \Theta_{t(2)}, \ldots, \Theta_{t\left(M_{\mathrm{T}}\right)}\right)$.

In what follows, we assume that $\hat{\boldsymbol{Y}}$ is an unbiased estimate of $\boldsymbol{Y}$, i.e. $\mathrm{M} \boldsymbol{\varepsilon}=0$, and that the measurement errors are small enough, so that the error analysis can be performed using the linearized version of the least squares method.

We additionally assume that all a priori fixed parameters are set to their true values (see however Sect. 2.3), that is $\tilde{\Theta}_{\mathrm{f}}=\Theta_{\mathrm{f}}$ for any $\Theta_{\mathrm{f}} \in \mathcal{F}$, then $\mathbf{M} \hat{\boldsymbol{\Theta}}=\boldsymbol{\Theta}$ and statistical properties of errors in parameter determination are completely characterized by the covariance matrix of errors $\mathrm{C}=\mathrm{D} \hat{\boldsymbol{\Theta}}$.

\subsection{Random errors and the relative figure of merit}

According to Kendall \& Stuart (1967, Chap. 19), the covariance matrix of errors $C$ is related to $\boldsymbol{Y}$ and $\boldsymbol{\Theta}$ by the following expression:

$\mathrm{C}(\boldsymbol{\Theta}, \mathcal{T}, \mathcal{N})=\left(\mathrm{A}^{\mathrm{T}}(\mathrm{D} \varepsilon)^{-1} \mathrm{~A}\right)^{-1}$

where $\mathrm{A}$ is the $M_{\mathrm{O}} \times\left(M_{\mathrm{T}}+M_{\mathrm{N}}\right)$ matrix with elements $A_{i j}=\frac{\partial Y_{i}}{\partial \Theta_{j}}$ for $\Theta_{j} \in \mathcal{T} \cup \mathcal{N}$ and $\varepsilon$ is the vector of experimental errors defined in Sect. 2.1.

The natural scalar characteristics of the precision of parameter determination for a given set $\mathcal{T}$ of target parameters is the following principal subdeterminant of the covariance matrix:

$C(\Theta, \mathcal{T}, \mathcal{N})=$

$$
=\operatorname{det}\left|\begin{array}{llll}
\mathrm{C}_{t(1) t(1)} & \mathrm{C}_{t(1) t(2)} & \ldots & \mathrm{C}_{t(1) t\left(M_{\mathrm{T}}\right)} \\
\mathrm{C}_{t(2) t(1)} & \mathrm{C}_{t(2) t(2)} & \ldots & \mathrm{C}_{t(2) t\left(M_{\mathrm{T}}\right)} \\
\vdots & \vdots & \ddots & \vdots \\
\mathrm{C}_{t\left(M_{\mathrm{T}}\right) t(1)} & \mathrm{C}_{t() t(2)} & \ldots & \mathrm{C}_{t\left(M_{\mathrm{T}}\right) t\left(M_{\mathrm{T}}\right)}
\end{array}\right|
$$

Geometrically, this subdeterminant is proportional to the hypervolume of the scattering ellipsoid in the space of target parameters. It depends not only on the physical model and errors of measurements, but also on the analytical form used for description of the model: two physically equivalent but mathematically different (e.g. interrelated by a reversible substitution of variables) analytical representations could yield entirely different values of $C(\boldsymbol{\Theta}, \mathcal{T}, \mathcal{N})$.

However, a pair of observational methods can well be compared if one makes use of the ratio

$$
R(\boldsymbol{\Theta}, \mathcal{T}, \mathcal{N})=\left(C^{\mathrm{I}}(\boldsymbol{\Theta}, \mathcal{T}, \mathcal{N}) / C^{\mathrm{II}}(\boldsymbol{\Theta}, \mathcal{T}, \mathcal{N})\right)^{1 / M_{\mathrm{T}}},
$$

where $C^{\mathrm{I}}$ and $C^{\mathrm{II}}$ are the subdeterminants calculated for the observation of the same object with instruments I and II. This ratio depends only on the physical model used, and the achieved precision on the definition of the model parameters. We shall call the quantity $R(\boldsymbol{\Theta}, \mathcal{T}, \mathcal{N})$, introduced by the Eq. (3), the "random error ratio", or the "relative figure of merit".

If the instruments are of the same kind, and differ from each other only in precision, the value $R(\boldsymbol{\Theta}, \mathcal{T}, \mathcal{N})$ is merely the ratio of observational errors. If the instruments are different, providing observables of different nature and number, and the target set consists of only one model parameter, the value $R(\boldsymbol{\Theta}, \mathcal{T}, \mathcal{N})$ is merely the ratio of resulting random errors in the parameter determination. However, in the general case of instruments of arbitrary kinds and multiparametric models, no simple ratio of errors exists, and the evaluation of relative merits can be done only using the quantity $R(\boldsymbol{\Theta}, \mathcal{T}, \mathcal{N})$ defined by Eq. (3).

This allows us to compare various observational techniques applied to objects described by various multiparametric models: equality $R(\boldsymbol{\Theta}, \mathcal{T}, \mathcal{N})<1$ means that instrument $I$ is better suited for determination of parameters from the target set than instrument II.

\subsection{Systematic errors induced by interpretation}

The fact that the description of an object by a multiparametric physical model is only an approximation to reality implies that we have to consider the robustness of the method, that is the stability of the results it yields with respect to deviations of the real situation from the model.

The present framework offers a way to obtain certain quantitative characteristics of the robustness. Indeed, let us consider a multiparametric model with $\mathcal{F} \neq \emptyset$. If $\tilde{\Theta}_{\mathrm{f}} \neq \Theta_{\mathrm{f}}$ for some $\Theta_{\mathrm{f}} \in \mathcal{F}$ then, in general, $\mathrm{M} \hat{\Theta}_{\mathrm{t}}-\Theta_{\mathrm{t}} \neq 0$ for $\Theta_{\mathrm{t}} \in \mathcal{T}$. That is, in addition to random errors of observational origin, the result is biased by systematic errors due to inaccurate interpretation. The value of that bias characterizes the robustness of the method with respect to deviations of $\tilde{\Theta}_{\mathrm{f}}$ from its true value.

In the linear approximation, $\mathrm{M}\left(\hat{\Theta}_{\mathrm{t}}-\Theta_{\mathrm{t}}\right)=\mathrm{S} \times\left(\tilde{\Theta}_{\mathrm{f}}-\right.$ $\Theta_{\mathrm{f}}$ ), where $\mathrm{S}$ is the $M_{\mathrm{T}} \times M_{\mathrm{F}}$ matrix with elements

$\mathrm{S}_{\mathrm{tf}}=\frac{\partial \Theta_{\mathrm{t}}}{\partial \Theta_{\mathrm{f}}}$

where $\Theta_{\mathrm{t}} \in \mathcal{T}, \quad \Theta_{\mathrm{f}} \in \mathcal{F}$. 
A comprehensive study of systematic errors requires a joint analysis of individual elements of the matrix $\mathrm{S}$ and uncertainties in model parameters from $\mathcal{F}$. In the present paper, we will develop a simplified approach providing semiquantitative indications concerning the relative robustness of different observational techniques.

Let us first define for each observational method under consideration the value

$U(\Theta, \mathcal{T}, \mathcal{F})=(C(\Theta, \mathcal{T}, \mathcal{F}) / C(\Theta, \mathcal{T}, \emptyset))^{1 / M_{\mathrm{T}}}$.

When the estimates for target parameters are uncorrelated with estimates for parameters from $\mathcal{F}$, equality $U=1$ takes place: the method is robust (with respect to deviations of specified form from the model). When such a correlation exists, $U$ exceeds unity; the closer the correlation, the larger its value, eventually implying poor robustness of the method.

Further, to compare the robustness of methods I and II with respect to inaccuracies in parameters from $\mathcal{F}$, we will define the "robustness ratio $S$ " as follows:

$S(\boldsymbol{\Theta}, \mathcal{T}, \mathcal{F})=U^{\mathrm{I}} / U^{\mathrm{II}}=R(\boldsymbol{\Theta}, \mathcal{T}, \mathcal{F}) / R(\boldsymbol{\Theta}, \mathcal{T}, \emptyset)$

where function $R$ is defined in Eq. (3). Note that once the values of $R(\Theta, \mathcal{T}, \mathcal{N})$, which are necessary for analysis of random errors, are found, comparison of robustness is straightforward.

\section{The relative figure of merit of OLBI vs. spectroscopy}

In this section we obtain the expressions for the elements of the covariance matrix $\mathbf{D} \hat{\boldsymbol{\Theta}}$ in the form that can be directly used to compare the OLBI and spectroscopy.

First, the measurement errors have to be specified. For this first trial application, the only considered source of random errors is the photon noise. Other sources of error, in particular those in OLBI arising from atmospheric seeing and calibrations of the modulation transfer function (see Roddier \& Léna 1984; Mourard et al. 1994) are important and have to be incorporated in future work.

Also, the spectral coverage and the spectral resolution have to be specified. We assume that they are the same for both kinds of observations; the covered spectral region is $\left[\lambda_{1}, \lambda_{2}\right]$, the spectral resolution is sufficiently high, i.e. the monochromatic intensity received from each point of the observed object remains nearly constant across each spectral channel. In other words, we assume that $\Delta \lambda$, the width of the spectral channels, in terms of velocity is lesser than the thermal velocity of the emitting atoms. For the P Cyg type envelopes this corresponds to $\Delta \lambda \lesssim 0.3 \AA$, which is easily satisfied with modern spectrographs. Then one can formally consider the convenient limit case $\Delta \lambda \rightarrow 0$. In this case, the resulting covariance matrices tend to a limit which is independent of the spectral resolution (cf. Eqs. (11) and (18)).

\subsection{Spectroscopy}

The physical quantity provided by spectroscopy is the flux density as a function of wavelength. In terms of observables, it is given by the vector $\hat{\boldsymbol{Y}}^{\mathrm{S}}$ with the components

$\hat{Y}_{i}^{\mathrm{S}}=\hat{N}_{i}, i=1,2, \ldots, L$,

where $\hat{N}_{i}$ is the number of photons recorded in the $i$-th spectral channel during the exposure, and $L$ is the number of channels. The random values $\hat{N}_{i}$ are related to the physical parameters of the object by

$\hat{N}_{i}=N_{i}+n_{i}$,

where

$N_{i}=F\left(\boldsymbol{\Theta}, \lambda_{i}\right) E \Delta_{i} \lambda$,

$F(\Theta, \lambda)$ is the monochromatic flux from the object at wavelength $\lambda, \Delta_{i} \lambda$ is the width of $i$-th spectral channel, and $n_{i}$ is the measurement error due to the photon noise, with the variance given by

$\mathrm{D} n_{i}=N_{i}$.

Finally the factor $E$ is given by

$E=A_{\text {eff }} t$,

where $A_{\text {eff }}$ is the instrument effective aperture and $t$ is the exposure time. Hereafter we suppose that $E^{\mathrm{S}}=E^{\mathrm{I}}$, where $E^{\mathrm{S}}$ and $E^{\mathrm{I}}$ pertain to the spectrometer and the interferometer respectively. Since the values $E^{\mathrm{S}}$ and $E^{\mathrm{I}}$ enter the final results only through the ratio $E^{\mathrm{S}} / E^{\mathrm{I}}$, we can for the sake of simplicity set $E^{\mathrm{S}}=E^{\mathrm{I}}=1$.

Thus, $\varepsilon_{i}^{\mathrm{S}}=n_{i}$ and the elements of the covariance matrix of measurement errors are given by

$\mathrm{M}\left(\varepsilon_{i}^{\mathrm{S}} \varepsilon_{j}^{\mathrm{S}}\right)=\left\{\begin{array}{ll}0 & \text { if } i \neq j \\ N_{i} & \text { if } i=j\end{array}\right.$.

Substituting Eqs. (6)-(10) into Eq. (1) and proceeding to the limit $\Delta_{i} \lambda \rightarrow 0$ we obtain:

$\left(\mathrm{C}^{\mathrm{S}}\right)_{\mathrm{pq}}^{-1}=\int_{\lambda_{1}}^{\lambda_{2}} \frac{1}{F(\boldsymbol{\Theta}, \lambda)} \frac{\partial F(\boldsymbol{\Theta}, \lambda)}{\partial \Theta_{\mathrm{p}}} \frac{\partial F(\boldsymbol{\Theta}, \lambda)}{\partial \Theta_{\mathrm{q}}} \mathrm{d} \lambda$.

For spherically symmetric objects considered in the present paper,

$F(\Theta, \lambda)=2 \pi \int_{0}^{\infty} I(\Theta, \lambda, p) \mathrm{d} p$

where $I(\Theta, \lambda, p)$ is the monochromatic intensity at the angular distance $p$ from the center of the object.

\section{2. $O L B I$}

We consider the case of a two-aperture OLBI with an adjustable baseline. The physical quantity eventually provided by OLBI measurements is the fringe visibility $V$ as a function of the baseline vector. The visibility $V$ is equal to the real part of the complex degree of coherence, and therefore is the Fourier transform of the brightness distribution in the focal plane by virtue of the Van Cittert - Zernicke theorem (e.g. Mariotti 1998, textbooks 
of Perina 1972, and Goodman 1985). However, the value estimated in practice is $V^{2}$. We refer the reader for details to the thorough discussion by Mourard et al. (1994). In the present analysis we consider the estimates $\hat{W}_{i}$ of the quantity

$W_{i}=W\left(\boldsymbol{\Theta}, \lambda_{i}, B\right)=V^{2}\left(\boldsymbol{\Theta}, \lambda_{i}, B\right)$,

where $i$ refers (as previously) to the $i$-th spectral channel, and $B$ is the projected baseline length of the interferometer. In the case of spherically symmetric objects the intensity distribution is circularly symmetric and $V$ is given by the normalized Hankel transform as follows (e.g. Bracewell 1978):

$V(\boldsymbol{\Theta}, \lambda, B)=\frac{2 \pi \int_{0}^{\infty} I(\boldsymbol{\Theta}, \lambda, p) J_{0}(k p) \mathrm{d} p}{F(\Theta, \lambda)}$,

where $J_{0}$ is the Bessel function, and $k=2 \pi B / \lambda$.

Statistical errors $w_{i}=\hat{W}_{i}-W_{i}$ obey the equations

$\mathrm{M}\left(w_{i} w_{j}\right)=\left\{\begin{array}{ll}0 & \text { if } i \neq j \\ \mathrm{D}\left(\hat{W}_{i}\right)=\frac{4 W_{i}}{N_{i}}\left(2-W_{i}\right) & \text { if } i=j\end{array}\right.$,

and

$\mathrm{M}\left(w_{i} n_{j}\right)=0$.

The validity of Eqs. (15) and (16) for $i \neq j$ is evident. The case $i=j$ is treated in Appendix A.

As a rule, the spectrum of the object, i.e. the values $\hat{N}_{i}$, is also recorded. Consequently, the vector of observables for the OLBI and the elements of its covariance matrix of errors are given by

$\hat{\boldsymbol{Y}}^{\mathrm{I}}=\left(\hat{N}_{1}, \hat{W}_{1}, \hat{N}_{2}, \hat{W}_{2}, \ldots, \hat{N}_{L}, \hat{W}_{L}\right)$

and

$\mathrm{M}\left(\varepsilon_{i}^{\mathrm{I}} \varepsilon_{j}^{\mathrm{I}}\right)=\left\{\begin{array}{ll}0 & \text { if } i \neq j \\ N_{k} & \text { if } i=j=2 k-1 \\ \frac{4 W_{k}}{N_{k}}\left(2-W_{k}\right) & \text { if } i=j=2 k\end{array}\right.$.

Substituting these expressions into Eq. (1), we obtain in the limit $\Delta_{i} \lambda \rightarrow 0$ that

$\left(\mathrm{C}^{\mathrm{I}}\right)_{\mathrm{pq}}^{-1}=\left(\mathrm{C}^{\mathrm{S}}\right)_{\mathrm{pq}}^{-1}+\int_{\lambda_{1}}^{\lambda_{2}} \frac{F}{4 W(2-W)} \frac{\partial W}{\partial \Theta_{\mathrm{p}}} \frac{\partial W}{\partial \Theta_{\mathrm{q}}} \mathrm{d} \lambda$,

where $\left(C^{S}\right)_{p q}^{-1}$ is defined in Eq. (11).

\subsection{The relative figure of merit}

As discussed in Sect. 2.2, the relative figure of merit of OLBI as compared to spectroscopy is the random error ratio:

$R^{\mathrm{IS}}(\boldsymbol{\Theta}, \mathcal{T}, \mathcal{N}, B)=C^{\mathrm{I}}(\boldsymbol{\Theta}, \mathcal{T}, \mathcal{N}, B) / C^{\mathrm{S}}(\boldsymbol{\Theta}, \mathcal{T}, \mathcal{N})$.

From Eqs. (18) and (19), it follows that $R^{\mathrm{IS}}<1$, which merely reflects the fact that the observables of spectroscopy constitute a subset of the OLBI observables. Consequently, in the adopted comparison, the statistical errors in parameter determination by OLBI are always less than those obtained when only spectroscopic data are used, if however the spectroscopic data are of equal precision.

As explained in Sect. 3.1, the Eq. (19) is obtained in the assumption that $E^{\mathrm{S}}=E^{\mathrm{I}}$. To compare a pair of instruments for which that equality does not hold, the RHS of Eq. (19) should be multiplied by $\left(E^{\mathrm{S}} / E^{\mathrm{I}}\right)^{1 / 2}$.

\section{Models of the P Cyg wind. Computed observables}

\subsection{Stellar parameters}

The star P Cyg (B1 Iape) is characterized by a high massloss rate and exhibits bright optical emission lines, formed in the dense and nearly fully ionized stellar wind. It belongs to the class of Luminous Blue Variables, a shortliving transition phase in the evolution of a massive star at the end of the hydrogen burning (see e.g. Humphreys \& Davidson 1994; Langer et al. 1994; Maeder 1997).

There were at least two strong reasons to choose P Cyg as the astrophysical object of the present study. First, this bright object, $V=4.8^{\mathrm{m}}$, is one of the best studied emission line stars. Although in this first trial we use simulated data, it is important to note that for $\mathrm{P}$ Cyg there exists a rich literature providing not only high quality spectroscopic data (e.g. Scuderi et al. 1994), but also interferometric data (Vakili et al. 1997), as well as thorough theoretical analysis (Drew 1985; Pauldrach \& Puls 1990). This ensures that the present work can be followed up by a practical application. Secondly, it happens that in the growing list of outflows known to be non-spherical (e.g. Wolf et al. 1998), P Cyg is an exception exhibiting spherical symmetry to a good degree of accuracy (Nota 1998), becoming clumpy only on short time and small flux scales (Taylor et al. 1991; Vakili et al. 1997; Nota 1998). This implies that the assumption of spherical symmetry of the envelope, used in the present work, is realistic. Note that it allows us to compute a model in a reasonable amount of time, and thus to explore a large domain of parametric space, which is a requirement for the evaluation results to be meaningful.

For the distance to the star $d$, its radius $R_{*}$ and the effective temperature $T_{\text {eff }}$ we adopted the following values: $d=1800 \mathrm{pc}, R_{*}=76.0 R_{\odot}, T_{\text {eff }}=20000 \mathrm{~K}$ (Lamers et al. 1983; Pauldrach \& Puls 1990). The spectrum of the star was assumed to be blackbody. Our study is limited to the hydrogen $\mathrm{H} \alpha$ line, which is the most prominent and the best studied feature in the spectrum of the star.

\subsection{The physical model of the envelope}

The mass distribution within a spherically symmetric and stationary outflow is described by its mass loss rate $\dot{M}$ and the outflow velocity $v$, which we assume to depend on radial distance $r$ in the following way:

$v(r)=v_{\mathrm{c}}+\left(v_{\infty}-v_{\mathrm{c}}\right)(1-1 / r)^{\alpha}$,

where $v_{\infty}$ is the terminal velocity of the wind, $v_{\mathrm{c}}$ is velocity of the wind at the base of the photosphere, and $\alpha$ is a dimensionless parameter that characterizes the rate at which the velocity approachs its asymptotic value at large $r$. The radial distance $r$ is measured in units of stellar radius $R_{*}$, while $\dot{M}, v_{\mathrm{c}}, v_{\infty}$, and $\alpha$ are parameters of a model. 
The temperature in the envelope is often assumed to be constant. However, as it was shown by Drew (1985), across the region of the $\mathrm{H} \alpha$ line formation $(R \lesssim 5)$ the temperature in the envelope can vary by as much as $6000 \mathrm{~K}$. Therefore, along with isothermal models, we also computed the emergent emission for non-isothermal models.

To keep a finite number of scalar parameters, the dependence of envelope temperature $T(r)$ on radial distance is approximated in the following manner: it is assumed that $T\left(r_{i}\right)=T_{i}$ for $r_{1}=1<r_{2}<\ldots<r_{M_{R}}$, that $T(r)$ is a linear function of $\log r$ at each interval $\left[r_{i}, r_{i+1}\right]$ for $0<i<M_{R}-1$, and that $T(r)=T_{M_{R}}$ for $r>r_{M_{R}}$. The values $r_{i}$ were fixed for each model of the family.

We consider only non-increasing temperature laws $T(r)$, that is $\Delta_{i} T \geq 0$ for $i=2, \ldots, M_{R}$, where $\Delta_{i} T=T_{i-1}-T_{i}$. Since the computations are organized in such a way that the model parameters vary independently on each other, it is the values $\Delta_{i} T$ that are used along with $T_{1}$ as the parameters defining the envelope temperature, and the vector of model parameters is given by

$\boldsymbol{\Theta}=\left(\alpha, \dot{M}, v_{\infty}, v_{\mathrm{c}}, T_{1}, \Delta_{2} T, \ldots, \Delta_{M_{R}} T\right)$.

\subsection{Source function and radiative transfer equation}

The "supersonic" Sobolev approximation is a well known efficient method of calculating line spectral profiles (e.g. Castor 1970). However, it would have a low accuracy if applied in the $\mathrm{P}$ Cyg case, for a noticeable fraction of $\mathrm{H} \alpha$ flux is emitted in the inner part of envelope, where the outflow velocity is comparable to that of sound. We adopted a mixed method computing the source function in the Sobolev approximation, and then finding the emergent intensities by the exact numerical solution of the transfer equation. As it was shown by Hamann (1981), the errors in line profiles computed in the original Sobolev approximation come mainly from calculations of the emergent intensities, whereas the source function is accurate for a wide range of physical conditions.

The choice of the model of the hydrogen atom was based on the fact that the regions of the envelope emitting the major fraction of the $\mathrm{H} \alpha$ flux are nearly completely ionized and opaque to Lyman continuum and $\mathrm{L} \alpha$, so that direct recombinations to and photoionizations from the ground level cancel out, and L $\alpha$ is saturated (Drew 1985). The populations of levels $n=2$ and $n=3$ are mainly defined by collisional and radiative transitions between these two levels, radiative ionizations due to stellar radiation, and radiative recombinations (including indirect) to the level $n=3$. We therefore adopted the three-level + continuum model of hydrogen atom.

The balance equations for $n_{2}$ and $n_{3}$, the number densities of hydrogen atoms respectively at levels 2 and 3 , take then the following form:

$$
\begin{aligned}
& n_{2}\left(C_{23}+I_{2}\right)-n_{3}\left(C_{32}+\beta_{32} A_{32}\right)=R_{2}, \\
& -n_{2} C_{23}+n_{3}\left(C_{32}+\beta_{32} A_{32}+I_{3}\right)=R_{3} \text {, }
\end{aligned}
$$

where $A_{32}$ is the spontaneous emission coefficient, $C_{23}$ and $C_{32}$ are collisional excitation and de-excitation coefficients for the transition, $I_{2}, I_{3}$ and $R_{2}, R_{3}$ are respectively ionization coefficients and recombination rates for the levels involved, and $\beta_{32}$ is the escape probability.

Since the hydrogen is nearly completely ionized, the values $C_{23}, C_{32}, R_{2}$ and $R_{3}$ can be considered independent of $n_{2}$ and $n_{3}$, the only source of non-linearity in Eqs. (20) being the terms containing $\beta_{23}$.

The escape probability $\beta_{32}$ is a rather complex function of the population of the lower level of the transition, so that commonly the Eqs. (20) are solved by iterations, which is, particularly the recalculation of the escape probability, by far the most time consuming operation.

To speed up computations, we developed a new approximate method for solving the equations of statistical equilibrium. It is based on the fact that, as shown in Appendix $\mathrm{B}$, the function $\beta_{32}\left(n_{2}\right)$ can be approximated with sufficiently good accuracy by a simple analytic expression as follows:

$\beta_{32}\left(n_{2}\right)=1 /\left(1+n_{2} / n_{\text {as }}\right)$,

where

$n_{\text {as }}=\frac{8 \pi}{\lambda^{3} A_{32}} \frac{g_{2}}{g_{3}}\left(\frac{1}{3} \frac{\mathrm{d} v(r)}{\mathrm{d} r}+\frac{2}{3} \frac{v(r)}{r}\right)$,

$g_{2}$ and $g_{3}$ are statistical weights of the levels, and $\lambda$ is the line wavelength.

Introducing the dimensionless variables

$P_{2}=n_{2} / n_{0}, \quad P_{3}=n_{3} /\left(n_{0} Q\right)$,

where

$n_{0}=\frac{R_{2}}{I_{2}}, \quad Q=\frac{g_{3}}{g_{2}} \exp \left(-\frac{E_{23}}{k T}\right)$,

and $E_{23}$ is the energy of transition, and substituting the approximation (21) into Eqs. (20) we obtain after some algebra that $P_{2}$ is the positive solution of the quadric equation

$a P_{2}^{2}+b P_{2}+c=0$,

where

$a=-\rho_{2} M S$,

$b=\left(\rho_{2} *\left(1-\beta_{0} *(\delta+S)\right)+M *\left(1-\beta_{0}\right)+\rho_{3}\right) P$,

$c=\beta_{0} *\left(\left(\rho_{2}+\rho_{3}\right) *(1+\delta)+M\right)=0$.

Here

$\rho_{i}=R_{i} /\left(A_{32} n_{0}\right) \quad$ for $\quad i=2,3$,

$M=\iota \rho_{2}^{2} \delta$,

$S=1+\iota Q$

$\beta_{0}=1 /\left(1+n_{0} / n_{\text {as }}\right)$.

and

$\iota=I_{3} / I_{2}$.

When $P_{2}$ is calculated, the value of $P_{3}$ can be found using the relation

$P_{3}=\frac{Q P_{2}+\rho_{3} \delta}{Q\left(\iota \rho_{2} \delta+\beta \delta+1\right)}$. 
The source function is then easily computed from level populations, which can be obtained using Eq. (23).

Integration of the transfer equation was performed using the code developed by Bertout (1984), who kindly provided it to the authors. In computing the line profile, the code assumes the envelope to be isothermal, so that its use for a non-isothermal case requires some comments. The envelope temperature enters the calculations at two points: (1) In calculating the source function, through coefficients $C_{23}$ and $C_{32}$ of Eq. (20). Since the Bertout code is applicable to arbitrary source function, variations in temperature does not cause any difficulties here. (2) In integrating the transfer equation, $T(r)$ enters the result through the local Doppler line width. Since we consider only the envelopes with relatively low temperature contrast $\left(T_{1}-T_{M_{R}}\right) / T_{1} \lesssim 0.4$, and dependence of the Doppler width on temperature is rather weak, the error induced by this isothermal code structure is still negligible.

\subsection{Computation of final results}

Once the intensity $I(\boldsymbol{\Theta}, \lambda, p)$ is computed, the observable quantities, i.e. the emergent flux $F(\boldsymbol{\Theta}, \lambda)$ and the visibility squared $W(\boldsymbol{\Theta}, \lambda, B)$ are obtained using Eqs. (12)-(14).

The relative figure of merit $R\left(\boldsymbol{\Theta}_{\mathrm{m}}, \mathcal{T}, \mathcal{N}, B_{j}\right)$ and the robustness ratio $S\left(\Theta_{\mathrm{m}}, \mathcal{T}, \mathcal{F}, B_{j}\right)$ are calculated in two stages, implemented as separate programs.

First, for the given grids of model parameters $\left\{\boldsymbol{\Theta}_{\mathrm{m}}: m=1, \ldots, N_{\mathrm{m}}\right\}$ and the projected baseline lengths $\left\{B_{j}: j=1, \ldots, N_{B}\right\}$, we calculate and store the matrices $\left(C^{S}\right)^{-1}$ and $\left(C^{I}\right)^{-1}$, defined in Eqs. (11) and (18). The defining parameters of the grids are entered as input data, the grid of models being constructed as the direct product of uniform grids for individual parameters.

The derivatives entering definitions of the matrices are approximated by finite differences. All the model parameters that are not constant on the grid are treated as adjustable, that is either target or nuisance. The distinction between those two types of parameters is irrelevant at this stage.

At the second stage, for given partitions of set $\mathcal{T} \cup \mathcal{N}$ of all adjustable parameters on the subsets $\mathcal{T}$ and $\mathcal{N}$, we compute the values of $R\left(\boldsymbol{\Theta}_{\mathrm{m}}, \mathcal{T}, \mathcal{N}, B_{j}\right)$ and $S\left(\boldsymbol{\Theta}_{\mathrm{m}}, \mathcal{T}, \mathcal{F}, B_{j}\right)$ (see Eqs. (3) and (5)) on the grid. In this way, the dependence of the results on $\mathcal{T}$ and $\mathcal{N}$ can be studied without repeating the time consuming physical modeling of the envelope.

\section{Results}

In total, the values $R(\boldsymbol{\Theta}, \mathcal{T}, \mathcal{N}, B)$ and $S(\boldsymbol{\Theta}, \mathcal{T}, \mathcal{F}, B)$ were calculated as a function of projected baseline length for various sets of target and nuisance parameters at more than $10^{5}$ points of the parametric space. Table 1 presents

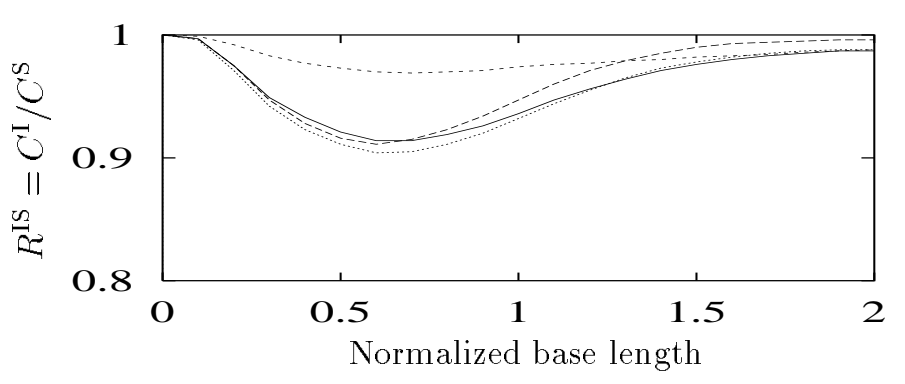

Fig. 1. Error ratio for isothermal model with $\alpha=3.94, \dot{M}=$ $1.4410^{-5} M_{\odot} \mathrm{yr}^{-1}, v_{\mathrm{c}}=32.8 \mathrm{~km} \mathrm{~s}^{-1}, T_{1}=12778 \mathrm{~K}, v_{\infty}=$ $200 \mathrm{~km} \mathrm{~s}^{-1}$, and the set of adjustable parameters $\mathcal{T} \cup \mathcal{N}=\{\alpha$, $\left.\dot{M}, v_{\mathrm{c}}, T_{1}\right\}$. Target set $\mathcal{T}=\{\alpha\}$ (solid line), $\{\dot{M}\}$ (dashed line), $\left\{T_{1}\right\}$ (short-dashed line), and $\left\{v_{\mathrm{c}}\right\}$ (dotted line)

the principal characteristics of the model grids. The ranges of variations of model parameters on the grids were chosen so as to include the values of parameters from earlier works on P Cyg cited in Sect. 4.1.

The main result of the present study is that the increase in accuracy provided by OLBI strongly depends on the number of free parameters in the model: it varies from almost negligible for models of low dimension to very significant for models of high dimension. Since in our case the boundary between "low" and "high" is nearly coincident with division into models with fixed and adjustable thermal structure, we discuss the results pertaining to those two classes of models separately.

\subsection{Models with fixed thermal structure}

Among our grids, the grid 1 most closely corresponds to the models often used in interpreting $\mathrm{H} \alpha$ observations of $\mathrm{P}$ Cyg stars, where $v_{\infty}$ is excluded from the set of adjustable parameters of the model (see e.g. Scuderi et al. 1994) and its value is taken from the analysis of absorption lines of metals in the ultraviolet (Casatella et al. 1979; Lamers et al. 1985).

The typical results for an individual point in the parametric space are illustrated by Fig. 1, where the random error ratio $R^{\mathrm{IS}}=C^{\mathrm{I}}(\boldsymbol{\Theta}, \mathcal{T}, \mathcal{N}, B) / C^{\mathrm{S}}(\boldsymbol{\Theta}, \mathcal{T}, \mathcal{N}, B)$ is plotted as a function of the normalized baseline length $B / B_{0}$ for various $\mathcal{T}$ and $\mathcal{N}$. Here $B$ is the projected baseline length, $B_{0}=\lambda / 2 \pi \delta$, and $\delta$ is the angular radius of the central star. For the adopted values of $R_{*}=76 R_{\odot}$, $d=1800 \mathrm{pc}$, and $\lambda=6265 \AA$ one gets $B_{0}=110 \mathrm{~m}$.

The principal features of dependence of $R^{\mathrm{IS}}$ on $B$ are independent of details of the physical model used and can be easily explained qualitatively. As shown in Sect. 3.3, inequality $R^{\mathrm{IS}}<1$ holds for all $0<B<\infty$. When the projected baseline length is very small, the interferometry does not provide any additional information as compared with the spectroscopy, for the object gets unresolved. Consequently, $R^{\mathrm{IS}} \rightarrow 1$ for $B \rightarrow 0$. In the opposite case of large $B$ the fringe contrast is close to 0 for all physically realistic intensity distributions and its dependence 
Table 1. Grids of models: the number of points in the parametric space $N_{\mathrm{m}}$; dimension, that is the number of varied parameters, of the grid $D$; the values of parameters that were fixed on the grid; and the ranges of variation for variable parameters

\begin{tabular}{|c|c|c|c|c|c|c|c|c|c|c|c|c|c|c|c|}
\hline $\begin{array}{l}\text { Grid } \\
\text { no. }\end{array}$ & $N_{\mathrm{m}}$ & $D$ & $\alpha$ & $\begin{array}{l}\dot{M} \\
M_{\odot} \mathrm{yr}^{-1}\end{array}$ & $\begin{array}{l}v_{\infty} \\
\mathrm{km} \mathrm{s}^{-1}\end{array}$ & $\begin{array}{l}v_{\mathrm{c}} \\
\mathrm{km} \mathrm{s}^{-1}\end{array}$ & $\begin{array}{l}T_{1} \\
\mathrm{~K} \\
\end{array}$ & $R_{2}$ & $\begin{array}{r}\Delta_{2} T \\
\mathrm{~K}\end{array}$ & $R_{3}$ & $\begin{array}{r}\Delta_{3} T \\
\mathrm{~K} \\
\end{array}$ & $R_{4}$ & $\begin{array}{r}\Delta_{4} T \\
\mathrm{~K} \\
\end{array}$ & $R_{5}$ & $\begin{array}{r}\Delta_{5} T \\
\mathrm{~K} \\
\end{array}$ \\
\hline 1 & 10000 & 3 & $\begin{array}{l}3.5 \\
4.5\end{array}$ & $\begin{array}{l}1.010^{-5} \\
2.010^{-5}\end{array}$ & 200 & $\begin{array}{l}15.0 \\
55.0\end{array}$ & $\begin{array}{l}11000 \\
15000\end{array}$ & & & & & & & & \\
\hline 2 & 10000 & 3 & $\begin{array}{l}3.5 \\
4.5\end{array}$ & $\begin{array}{l}1.010^{-5} \\
2.010^{-5}\end{array}$ & 200 & $\begin{array}{l}15.0 \\
55.0\end{array}$ & $\begin{array}{l}11000 \\
15000\end{array}$ & 2.0 & 1300 & 3.4 & 2100 & 5.0 & 200 & 10.0 & 3000 \\
\hline 3 & 32805 & 4 & $\begin{array}{l}3.5 \\
4.5\end{array}$ & $\begin{array}{l}1.010^{-5} \\
2.010^{-5}\end{array}$ & $\begin{array}{l}150 \\
250\end{array}$ & $\begin{array}{l}15.0 \\
55.0\end{array}$ & $\begin{array}{l}11000 \\
15000\end{array}$ & & & & & & & & \\
\hline 4 & 15625 & 6 & $\begin{array}{l}3.5 \\
4.5\end{array}$ & $\begin{array}{l}1.010^{-5} \\
2.010^{-5}\end{array}$ & 200 & $\begin{array}{l}15.0 \\
55.0\end{array}$ & $\begin{array}{l}11000 \\
15000\end{array}$ & 2.0 & $\begin{array}{r}0 \\
1300\end{array}$ & 5.0 & $\begin{array}{r}0 \\
2300\end{array}$ & & & & \\
\hline 5 & 46656 & 6 & $\begin{array}{l}3.25 \\
4.25\end{array}$ & $\begin{array}{l}1.010^{-5} \\
2.010^{-5}\end{array}$ & 200 & $\begin{array}{l}15.0 \\
55.0\end{array}$ & $\begin{array}{l}11000 \\
15000\end{array}$ & 2.0 & $\begin{array}{r}0 \\
1300\end{array}$ & 3.4 & $\begin{array}{r}0 \\
2100\end{array}$ & & & & \\
\hline 6 & 6561 & 8 & $\begin{array}{l}3.5 \\
4.5\end{array}$ & $\begin{array}{l}1.010^{-5} \\
2.010^{-5}\end{array}$ & 200 & $\begin{array}{l}15.0 \\
55.0\end{array}$ & $\begin{array}{l}11000 \\
15000\end{array}$ & 2.0 & $\begin{array}{r}0 \\
1300\end{array}$ & 3.4 & $\begin{array}{r}0 \\
2100\end{array}$ & 5.0 & $\begin{array}{r}0 \\
200\end{array}$ & 10.0 & $\begin{array}{r}0 \\
3000\end{array}$ \\
\hline 7 & 65536 & 8 & $\begin{array}{l}3.5 \\
4.5\end{array}$ & $\begin{array}{l}1.010^{-5} \\
2.010^{-5}\end{array}$ & 200 & $\begin{array}{l}15.0 \\
55.0\end{array}$ & $\begin{array}{l}11000 \\
15000\end{array}$ & 2.0 & $\begin{array}{r}0 \\
1300\end{array}$ & 3.0 & $\begin{array}{r}0 \\
2100\end{array}$ & 4.0 & $\begin{array}{r}0 \\
200\end{array}$ & 5.0 & $\begin{array}{r}0 \\
3000\end{array}$ \\
\hline 8 & 6561 & 8 & $\begin{array}{l}4.0 \\
4.5\end{array}$ & $\begin{array}{l}1.510^{-5} \\
2.310^{-5}\end{array}$ & $\begin{array}{l}180 \\
220\end{array}$ & 20.0 & $\begin{array}{l}14000 \\
16000\end{array}$ & 2.0 & $\begin{array}{r}0 \\
1000\end{array}$ & 3.0 & $\begin{array}{r}0 \\
1000\end{array}$ & 4.0 & $\begin{array}{r}0 \\
1000\end{array}$ & 5.0 & $\begin{array}{r}0 \\
1000\end{array}$ \\
\hline 9 & 19683 & 9 & $\begin{array}{l}3.5 \\
4.5\end{array}$ & $\begin{array}{l}1.510^{-5} \\
2.310^{-5}\end{array}$ & $\begin{array}{l}220 \\
300\end{array}$ & $\begin{array}{l}15.0 \\
25.0\end{array}$ & $\begin{array}{l}16000 \\
18000\end{array}$ & 2.0 & $\begin{array}{r}0 \\
1300\end{array}$ & 3.0 & $\begin{array}{r}0 \\
2100\end{array}$ & 4.0 & $\begin{array}{r}0 \\
200\end{array}$ & 5.0 & $\begin{array}{r}0 \\
3000\end{array}$ \\
\hline
\end{tabular}

on model parameters is impossible to measure because of observational errors. That is, in that case the interferometry again provides no additional information and $R^{\mathrm{IS}} \rightarrow 1$ for $B \rightarrow \infty$.

It follows from the above stated general properties of $R^{\mathrm{IS}}$ that for any $\mathcal{T}$ and $\mathcal{N}$ it has a (possibly non-unique) minimum at a certain finite values of $B$. The location of the minimum indicates the projected baseline length at which interferometric observations are most informative, and the corresponding value of $R^{\mathrm{IS}}$ characterize to what degree the interferometry can reduce the errors in model parameters determination as compared to spectroscopy.

Let us note that it may not be correct to compare the merits of the methods using values of $R^{\mathrm{IS}}$ obtained for an isolated point or even for a few arbitrarily chosen points of parametric space. Since the model parameters are not known a priori, it is necessary to use integral characteristics describing the behaviour of $R^{\mathrm{IS}}$ on the whole grid. We will use the following three values calculated as a function of $B_{j} / B_{0}$ for various $\mathcal{T}$ and $\mathcal{N}$ :

$$
\begin{aligned}
& R_{\min }\left(\mathcal{T}, \mathcal{N}, B_{j}\right)=\min _{1 \leq m \leq N_{\mathrm{m}}} R^{\mathrm{IS}}\left(\Theta_{m}, \mathcal{T}, \mathcal{N}, B_{j}\right) \\
& R_{\max }\left(\mathcal{T}, \mathcal{N}, B_{j}\right)=\max _{1 \leq m \leq N_{\mathrm{m}}} R^{\mathrm{IS}}\left(\Theta_{m}, \mathcal{T}, \mathcal{N}, B_{j}\right) \\
& P_{\text {opt }}\left(\mathcal{T}, \mathcal{N}, B_{j}\right)=N_{\text {opt }} / N_{\mathrm{m}},
\end{aligned}
$$

where $N_{\text {opt }}$ is the number of points on the grid for which function $R^{\mathrm{IS}}(\Theta, \mathcal{T}, \mathcal{N}, B)$ reaches its minimum at $B=B_{j}$.
The function defined in Eq. (26) is immediately related to the problem of optimal choice of the baseline length: for given $\mathcal{T}$ and $\mathcal{N}$, the higher the value $P_{\text {opt }}(\mathcal{T}, \mathcal{N}, B)$, the higher the probability that the interferometric observations at the baseline length $B$ would yield the most accurate model parameters.

The dependence of $R_{\mathrm{min}}$ and $P_{\mathrm{opt}}$ on the normalized baseline length and set of target parameters for grid 1 is shown in Fig. 2. For this grid, the value of $R_{\max }$ is close to unity for all $\mathcal{T}$ and $\mathcal{N}$ and is not shown in the plot. It can be easily seen that for this grid of models OLBI provides only a relatively small reduction of the random error in parameter determination as compared with spectroscopy. This conclusion remains also valid if we add the terminal velocity $v_{\infty}$ to the set of adjustable parameters (grid 3 ).

As $R^{\mathrm{IS}}(\boldsymbol{\Theta}, \mathcal{T}, \mathcal{N}, B) \approx 1$ for all $\mathcal{T}$ and $\mathcal{N}$, the same approximate equality is evidently valid for the robustness ratio $S(\boldsymbol{\Theta}, \mathcal{T}, \mathcal{F}, B)=R(\boldsymbol{\Theta}, \mathcal{T}, \mathcal{F}, B) / R(\boldsymbol{\Theta}, \mathcal{T}, \emptyset, B)$, which indicates (see Sect. 2.3) that for models with a priori fixed thermal structure, interferometric data will not reduce appreciably the level of systematic errors.

\subsection{Models with adjustable thermal structure}

The number of possible target sets is an exponentially increasing function of the dimension $D$ of the model parameter space, so that when the values $\Delta_{i} T$ are added to the set 


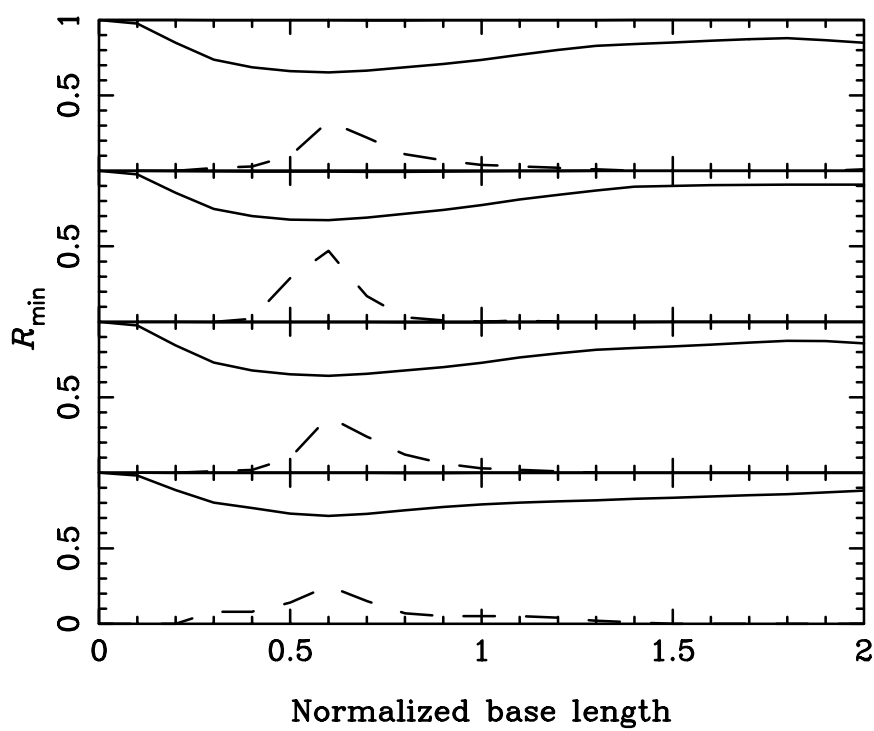

Fig. 2. The lower limit of random error ratio $R_{\min }$ (solid line) and distribution of optimal baseline lengths $P_{\text {opt }}$ (dashed lines, plotted in an arbitrary scale) for grid 1 . The set of adjustable parameters $\mathcal{T} \cup \mathcal{N}=\left\{\alpha, M, v_{\mathrm{c}}, T_{1}\right\}$, target parameters are indicated on the plots

of unknowns, it becomes impossible to make an exhaustive presentation of even the integral results, i.e. the functions $R_{\min }(\mathcal{T}, \mathcal{N}, B), R_{\max }(\mathcal{T}, \mathcal{N}, B)$, and $P_{\text {opt }}(\mathcal{T}, \mathcal{N}, B)$.

In Fig. 3, we show the dependence of $R_{\min }, R_{\max }$, and $P_{\text {opt }}$ on the number of nuisance parameters for one of our simplest grids of models with adjustable thermal structure, the target set consisting of one parameter, the mass loss rate $\dot{M}$. From many points of view, it is the most important parameter of the stellar wind, characterizing both its effect on the evolution of the star and the influence of the outflowing matter on the surrounding interstellar medium.

As it can be seen, the lower limit of the random error ratio $R_{\min }$ rapidly decreases as the number of adjustable temperature parameters increases, so that when the thermal structure of the envelope becomes a nuisance parameter, interferometry can reduce the random error in $\dot{M}$ by as much as an order of magnitude.

Note, that the decrease in $R_{\min }$ is caused by the fact that parameters $\Delta_{i} T$ are adjustable, rather then by deviation from isothermicity. This can be seen from the upper two plots in Fig. 3, where $R_{\min }>0.8$ for a grid containing a high proportion of models with a noticeable temperature gradient, and is also supported by the results obtained for grid 2, which differs from grid 1 only in that its thermal structure approximates that of Drew's model B, which corresponds to $\alpha=4, v_{\mathrm{c}}=15 \mathrm{~km} \mathrm{~s}^{-1}, v_{\infty}=300 \mathrm{~km} \mathrm{~s}^{-1}$, and $\dot{M}=1.510^{5} M_{\odot} \mathrm{yr}^{-1}$ in our notations, instead of being isothermal.

For other single-parameter target sets the dependencies of $R_{\min }$ and $R_{\max }$ on $\mathcal{N}$ are qualitatively the same. Figure 4 displays the results for the most important case

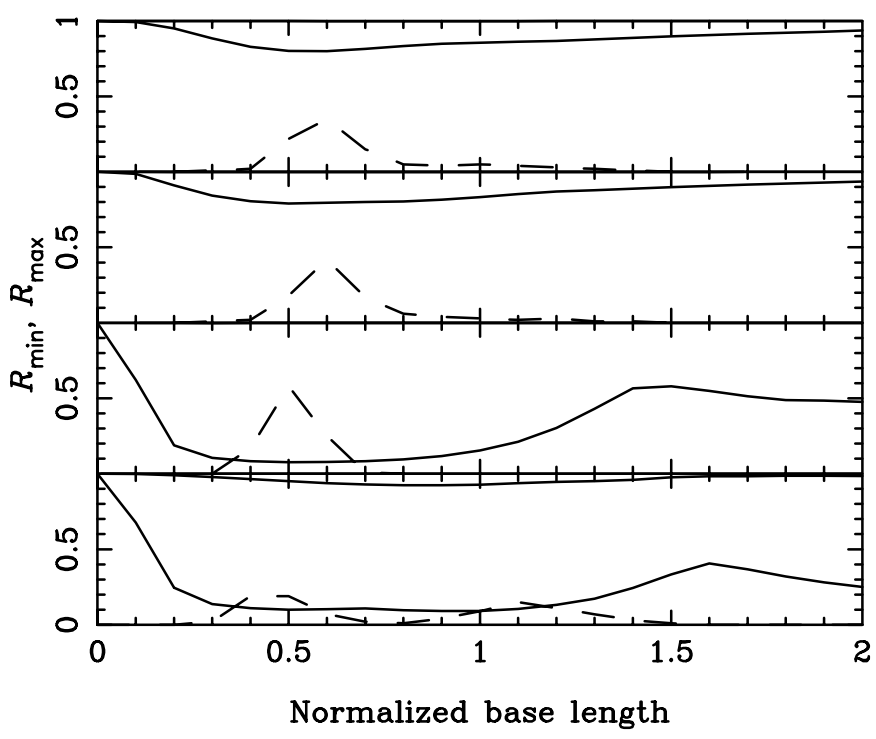

Fig. 3. The lower and upper limits of random error ratio (solid lines, $R_{\max }$ differs noticeably from unity only in the bottom plot) and distribution of optimal baseline lengths $P_{\mathrm{opt}}$ (dashed lines, plotted in an arbitrary scale) for grid 5 . Target set $\mathcal{T}=\{\dot{M}\}$, nuisance parameters are indicated on the plots

when all the parameters of the model except for $v_{\infty}$ are adjustable. As one can see, again, in favorable circumstances interferometry can increase accuracy by an order of magnitude. In contrast to $R_{\min }$, the value $R_{\max }$ is close to unity for all projected baseline lengths and combinations of sets $\mathcal{T}$ and $\mathcal{N}$ that we have studied. This signifies that for any projected baselength there exists a combination of model parameters for which interferometry practically do not provide reduction in error.

It is interesting to notice that for target sets containing several parameters (see Fig. 5), the influence of individual parameters on $R_{\min }$ and $R_{\max }$ to some extent averages out: $R_{\min }$ is systematically higher, and $R_{\max }$ is systematically lower than the corresponding values for individual parameters. For this reason, when more than one model parameter is to be determined, interferometry at a single projected baselength is unlikely to yield overall gain in accuracy in excess of a factor of two even at optimal baselines.

We studied further the effect of increasing the number of sublayers $M_{R}$, which permits us to investigate the influence of fine details of the thermal structure of the envelope. This appears to be justified because Drew (1985) showed that in certain cases this structure is quite complex, and the gradient of $\log T(r)$ varies strongly with radial distance. Consequently, a model aiming to closely approximate the realistic envelope should be parameterized using a large $M_{R}$ (see Sect. 4.1) and hence requires computations on grids of high dimension $D$.

Since the number of mesh points of the grid depends on $D$ exponentially, for computations based on such complex models (grids 5-9 from Table 1) the limited computer 


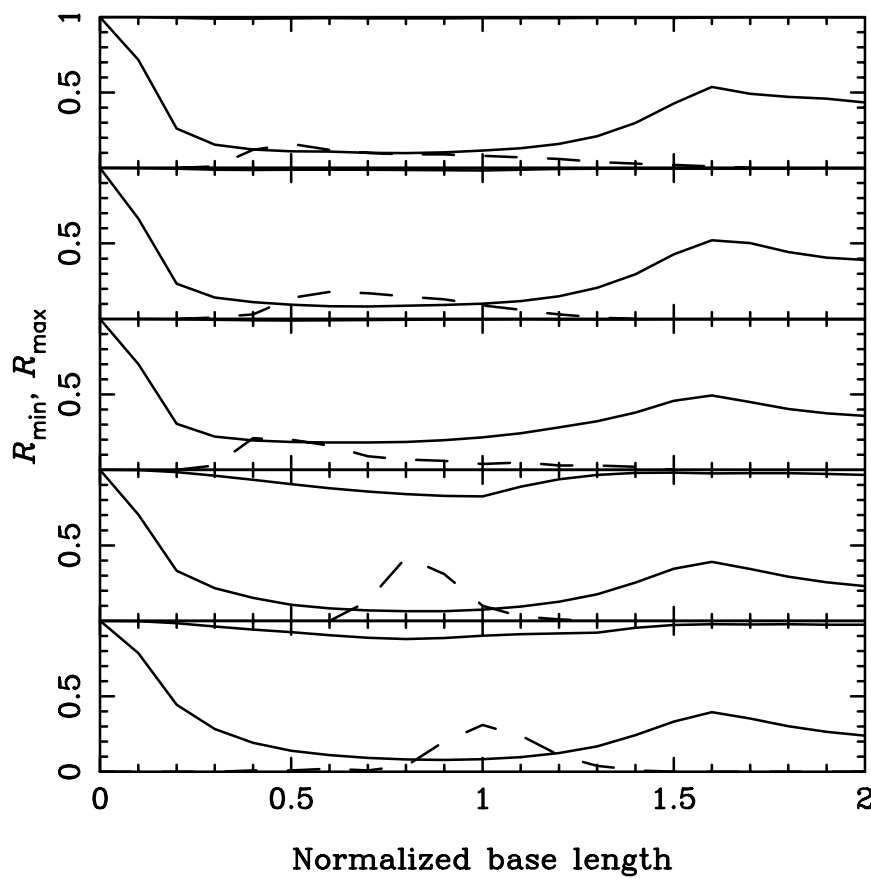

Fig. 4. The lower and upper limits of random error ratio (solid lines) and distribution of optimal baseline lengths $P_{\text {opt }}$ (dashed lines) for grid $5, \mathcal{T} \cup \mathcal{N}=\left\{\alpha, \dot{M}, v_{\mathrm{c}}, T_{1}, \Delta_{2} T, \Delta_{3} T\right\}$, and target sets indicated on the plots

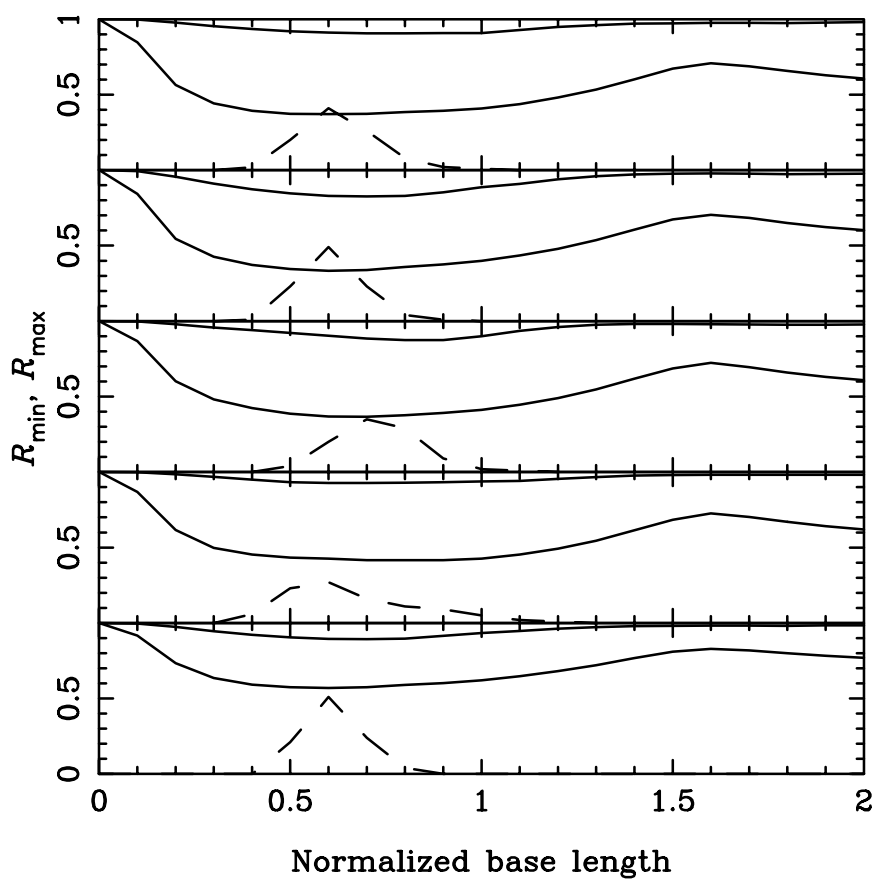

Fig. 5. The lower and upper limits of random error ratio (solid lines) and distribution of optimal baseline lengths $P_{\text {opt }}$ (dashed lines) for grid $5, \mathcal{T} \cup \mathcal{N}=\left\{\alpha, \dot{M}, v_{\mathrm{c}}, T_{1}, \Delta_{2} T, \Delta_{3} T\right\}$, and target sets comprising several parameters (indicated on the plots)

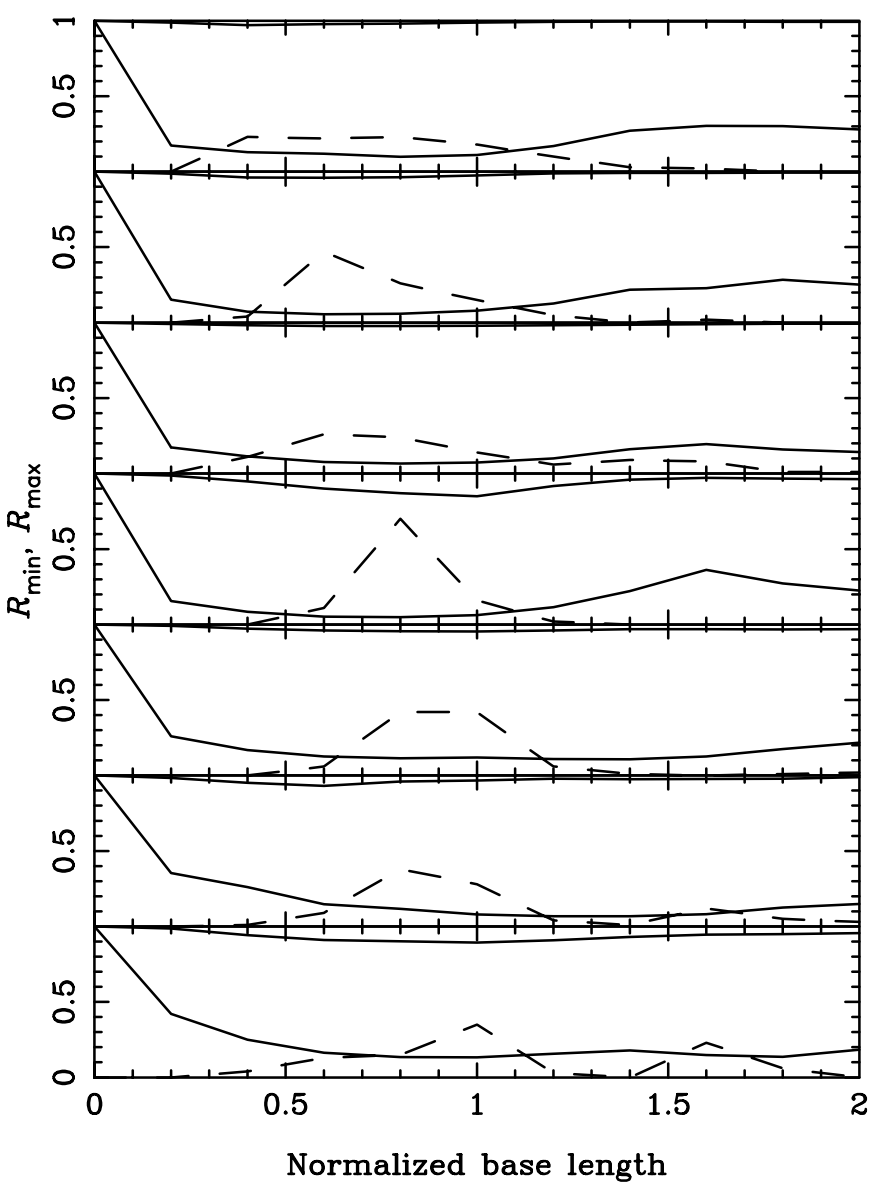

Fig. 6. The lower and upper limits of random error ratio (solid lines) and distribution of optimal baseline lengths $P_{\text {opt }}$ (dashed lines) for grid $7, \mathcal{T} \cup \mathcal{N}=\left\{\alpha, \dot{M}, v_{\mathrm{c}}, T_{1}, \Delta_{2} T, \Delta_{3} T, \Delta_{4} T, \Delta_{5} T\right\}$, and target sets consisting of a single parameter (indicated on the plots)

resources dictate sparser mesh points along each axis of the parameter space and a tuning of parameters controlling the computational process in such a way as to increase the speed of computation at the expense of precision.

Although numerically less precise than computations for simpler models, our results for more realistic grids indicate that the conclusions derived for low $M_{R}$ can be safely extrapolated for higher $M_{R}$. This point is illustrated in Fig. 6 showing the results for single-parameter target sets for grid 7. As can be seen in Figs. 3-6 and is confirmed by other results not presented here, the distribution of optimal projected baseline lengths in the majority of cases has a maximum in the range $0.4-0.8$ in dimensionless units. In some cases this maximum shifts to higher baseline lengths, but this occurs only for a very particular choice of $\mathcal{T}$ that correspond to measurements of wind temperature gradient at distances of several stellar radii from the star with all other model parameters considered nuisance (see e.g. bottom plots in Figs. 4 and 6). Thus, the aforementioned range, corresponding to $45-90 \mathrm{~m}$ in linear scale, can be recommended as the optimal choice for interferometric 


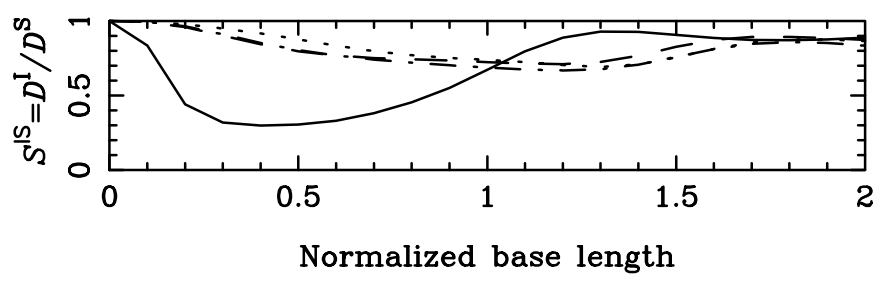

Fig. 7. Robustness ratio for the model $\alpha=4.00$, $\dot{M}=1.510^{-5} M_{\odot} / \mathrm{yr}, v_{\mathrm{c}}=35.0 \mathrm{~km} \mathrm{~s}^{-1}, T_{1}=13000 \mathrm{~K}$, $\Delta_{2} T=650 \mathrm{~K}, \Delta_{3} T=1050 \mathrm{~K}, \Delta_{4} T=100 \mathrm{~K}, \Delta_{5} T=1500 \mathrm{~K}$ from grid 6 and $\mathcal{T}=\{\dot{M}\}$. Set of fixed parameters $\mathcal{F}=\left\{\alpha, v_{\mathrm{c}}\right.$, $\left.T_{1}, \Delta_{2} T\right\}$ (solid line), $\left\{\alpha, v_{\mathrm{c}}, T_{1}, \Delta_{2} T, \Delta_{3} T\right\}$ (dashed line), $\left\{\alpha, v_{\mathrm{c}}, T_{1}, \Delta_{2} T, \Delta_{3} T, \Delta_{4} T\right\}$ (dash-dotted line), and $\left\{\alpha, v_{\mathrm{c}}\right.$, $\left.T_{1}, \Delta_{2} T, \Delta_{3} T, \Delta_{4} T, \Delta_{5} T\right\}$ (dotted line)

observations aimed at determination of global characteristics of the P Cyg wind.

The typical dependence of the robustness ratio $S^{\mathrm{IS}}(\boldsymbol{\Theta}, \mathcal{T}, \mathcal{N}, B)=U^{\mathrm{I}} / U^{\mathrm{S}}$ on the baseline length and the set of fixed parameters for a model with adjustable thermal structure is shown in Fig. 7. At nearly all points of parametric space $S^{\mathrm{IS}}<1$, and $S^{\mathrm{IS}}$ is generally lower when the set of fixed parameters $\mathcal{F}$ includes the values $\Delta_{i} T$. For a small fraction of models and for short projected baselines where interferometry can not significantly reduce random error, the value $S^{\mathrm{IS}}>1$ by a negligible amount.

Thus, our results indicate that if the baseline length and the model used for interpretation are chosen in such a way as to reduce random errors, OLBI also appears able to reduce the systematic errors.

\section{Conclusions}

Using the theory of model parameter determination, we developed a general method which permits a quantitative comparison of observational techniques and optimization of complex observations through the relative figure of merit, defined as a generalization of the ratio of random errors of model parameters.

The method was applied to compare the outcome of OLBI and classical spectroscopic observations, both used to determine the parameters of the outflow of the P Cyg star. The observable quantities were computed using an efficient radiative transfer code and realistic grids of the envelope model. The OLBI and spectroscopic measurements errors were simulated assuming that the only source of errors is the counting statistics, i.e. we considered a nearly-ideal interferometer. Other main assumptions and simplifications of the present work were as follows: (1) the P Cyg wind is stationary and spherically symmetric; (2) the only OLBI observable considered here is the visibility modulus; (3) only the hydrogen $\mathrm{H} \alpha$ line is considered.

The main conclusions of the present work are as follows:
1. A meaningful evaluation of the OLBI vs. spectroscopy relative figure of merit requires exploration of a large domain of the model parametric space (about $10^{5}$ points computed in the present work);

2. If the P Cyg outflow can be accurately described by a simple model of a stationary spherically symmetric isothermal envelope, then the use of interferometry does not substantially reduce the random error in the model parameter determination;

3. If the P Cyg outflow requires more complex models, e.g. with a priori unknown dependence of the envelope temperature on radial distance, use of a nearly-ideal interferometer can reduce errors in model parameters by an order of magnitude as compared to the spectrometer of the same collecting power and noise level;

4. The study of the relative robustness, i.e. the stability of the obtained model parameter determination with respect to systematic biases in the model, indicates that when the OLBI and the physical model reduce random errors, they also tend to reduce the systematic error;

5. The optimal projected baseline lengths for observation of P Cyg with a nearly ideal interferometer lie in the range $45-90 \mathrm{~m}$;

6. If several model parameters are to be determined simultaneously, the OLBI observations at a single projected baseline cannot provide a substantial error reduction.

\section{Final remarks}

In this section we discuss to what extent our results are relevant for real observations, and how the methods developed here could be generalized to more complex cases.

\subsection{Additional sources of measurement errors}

Although general formalism presented in Sect. 2 remains valid for any source of errors provided that the linearized least-square method is applicable, Eqs. (11) and (18) need to be modified if the accuracy of measurements is not shot noise limited.

In the simplest case of uncorrelated errors in spectral channels, this modification reduces to multiplication of the integrands in Eqs. (11) and (18) by corresponding ratios (shot noise error)/(total error). If these ratios are nearly constant across the observed spectral range, then after appropriate scaling our results can be directly applied to real observations.

The assumption of uncorrelated errors may be invalid for interferometric observations, the observables $\hat{W}_{i}$ being obtained as a result of much more complicated processing of raw observational data (cf. Mourard et al. 1994) then that considered in Appendix A. This point must be analysed further, and in future studies, it may be necessary to use directly Eq. (1) instead of Eq. (18). 
In any case, a generalization on arbitrary measurement errors could be easily incorporated into the used code with no considerable increase in the required computer resources.

\subsection{Observation in several spectral lines}

The theory developed in Sect. 2 can be applied to the case of multiple observed lines without any modifications. Formally, it reduces to changing integration domains in Eqs. (11) and (18).

Rapid evaluation of source function, on the contrary, would require a detailed study and development of efficient methods specific to each spectral line involved, because different lines can be emitted in different regions of the envelope and originate from different physical processes.

\subsection{Multi-baseline OLBI of spherically symmetric objects}

The method used here can be generalized to visibility measurements done simultaneously at more than one baseline: the only modification required is the replacement of RHSes of Eq. (18) by the sum of corresponding expressions over a given set of projected baseline lengths, the directions of the baselines being unimportant because of circular symmetry of intensity distribution. This can be done by modifying only the code performing the second stage of calculations (see Sect. 4.4) and using the data prepared for the single-baseline case during the most time consuming first stage of computation.

\subsection{Asymmetric brightness distribution and information on fringe phase}

Although time-averaged structure of P Cyg wind can be considered spherically symmetric, polarimetric (Taylor et al. 1991) and interferometric (Vakili et al. 1997) observations revealed the existence of time-dependent "clumpy" structures in the envelope, giving rise to deviations from spherical symmetry.

In this case, measurements of the fringe phase can yield important information. This contrasts with the spherically symmetric case, where the fringe phase is irrelevant to physical modeling of the object.

On the other hand, a unique determination of model parameters using only spatially unresolved spectroscopy appears impossible for the complex physical models resulting in two-dimensional brightness distributions, so that even the formulation of the comparison problem for spectroscopy and interferometry can hardly be done. The choice of the optimal set of baseline lengths for interferometry still remains the important problem, and the method developed here can be used for such an optimization after certain modifications.
First, the fringe phases (or observable linear combinations thereof) should be included in the set of observables defined in Eq. (17), and Eq. (18) should be modified accordingly. This would require comprehensive error analysis depending on the particular method used for extracting phase information from measurements.

Second, errors in parameter determination for observations performed with interferometer configurations $B_{1}$ and $B_{2}$ (here $B_{1}$ and $B_{2}$ denote corresponding sets of simultaneously used projected baseline lengths) can be compared using the ratio $C^{\mathrm{I}}\left(\boldsymbol{\Theta}, \mathcal{T}, \mathcal{N}, B_{1}\right) / C^{\mathrm{I}}\left(\boldsymbol{\Theta}, \mathcal{T}, \mathcal{N}, B_{2}\right)$ instead of $R^{\mathrm{IS}}(\boldsymbol{\Theta}, \mathcal{T}, \mathcal{N}, B)$.

Acknowledgements. The authors are grateful to A. Labeyrie, D. Mourard and F. Vakili for encouraging discussion at the beginning of this project. We thank C.Bertout who provided the code for computation of the emergent intensities. MSB is grateful to the staff of Observatoire de Grenoble for their hospitality during his stay in Grenoble. Suggestions by the referee P. Stee and correctons of English by S. McKenzie helped to improve the article, we are thankful to them. This research made use of SIMBAD astronomical database, created and maintained by the CDS, Strasbourg, and NASA's Astrophysics Data System Abstract Service. Part of this work was supported by CNRS through the grants PICS No. 194 "France - Russie, Astronomie à haute résolution angulaire", GdR "Milieux circumstellaires", and GdR and PN "Haute résolution angulaire en Astronomie".

\section{Appendix A: Computation of $\mathrm{D} w_{i}$ and $\mathrm{M}\left(n_{i} w_{i}\right)$}

In this Appendix, we compute the non-trivial elements of the covariance matrix of errors used in Sect. 3.2. Since we consider here a single spectral channel, the index $i$ will be omitted everywhere. Thus, the values $N, \hat{N}, n, W, \hat{W}$, and $w$ in this Appendix are identical with the values denoted as $N_{i}, \hat{N}_{i}, n_{i}, W_{i}, \hat{W}_{i}$, and $w_{i}$ respectively in Sect. 2 .

In practice, the error in $\hat{W}$ depends on too many details of experimental techniques, atmospheric conditions and data processing methods to be analyzed in general form (Mourard et al. 1994). The present analysis is restricted to a highly idealized situation. Namely, as well as in Sect. 3.1, the only source of measurement errors taken into account is the photon shot noise. We assume that the static fringes are detected by counting the photons in nonintersecting channels that cover in total the interval of length $Z$ of optical path difference (OPD). If $z$ is the $\mathrm{OPD}$, which is measured here in units of $\lambda / 2 \pi$, and $F(z)$ is the photon counting rate per unit $z$, then

$F(z)=N\left(1+V \cos \left(z+z_{0}\right)\right) / Z$,

where $z_{0}$ is unknown fringe phase shift and $N$ is proportional to the total received flux.

If we define

$C=2 \int_{0}^{Z} F(z) \cos z \mathrm{~d} z$ 
and

$S=2 \int_{0}^{Z} F(z) \sin z \mathrm{~d} z$,

then assuming that $Z \gg 1$ and neglecting the terms that decrease as $1 / Z$ for $Z \rightarrow \infty$ we obtain that

$N=\int_{0}^{Z} F(z) \mathrm{d} z$

and

$V^{2}=\left(C^{2}+S^{2}\right) / N^{2}$

An estimate for $W=V^{2}$ can be constructed by replacing the values in the RHS of Eq. (A5) by their estimates that can be easily deduced from Eqs. (A2)-(A4). If the interval of OPDs covered by measurements is divided in $M$ subintervals of length $\Delta_{p} z$ and $\Delta_{p} z \ll 1$ for $p=1, \ldots, M$, then an estimate $\hat{W}$ of $W$ based upon the number of photons received in each of these subintervals is given by

$\hat{W}=\left(\hat{C}^{2}+\hat{S}^{2}\right) / \hat{N}^{2}$,

where $\hat{N}=\sum_{p=1}^{M} \hat{N}_{\mathrm{p}}, \hat{N}_{\mathrm{p}}$ is the number of photons received in the $p$-th subinterval, $\hat{C}=2 \sum_{p=1}^{M} \hat{N}_{\mathrm{p}} \cos z_{\mathrm{p}}$, and $\hat{S}=2 \sum_{p=1}^{M} \hat{N}_{\mathrm{p}} \sin z_{\mathrm{p}}$.

If we assume further that $\hat{W}$ as a function of $n_{\mathrm{p}}=$ $\hat{N}_{\mathrm{p}}-N_{\mathrm{p}}$ can be linearized in the vicinity of zero and take into account that $\mathrm{M}\left(n_{\mathrm{p}}\right)=0$ and $\mathrm{M}\left(n_{\mathrm{p}} n_{\mathrm{q}}\right)=\delta_{\mathrm{pq}} N_{\mathrm{p}}$, then after some algebra we obtain for the statistical characteristics of $\hat{W}$

$$
\begin{gathered}
\mathrm{D}(w)=\mathrm{M}\left(\left(\sum_{p=1}^{M} \frac{\partial W}{\partial N_{\mathrm{p}}} n_{\mathrm{p}}\right)^{2}\right)=\frac{4 W}{N}(2-W), \\
\mathrm{M}(n w)=\mathrm{M}\left(\left(\sum_{p=1}^{M} \frac{\partial W}{\partial N_{\mathrm{p}}} n_{\mathrm{p}}\right)\left(\sum_{q=1}^{M} n_{\mathrm{q}}\right)\right)=0,
\end{gathered}
$$

which proves the validity of Eqs. (15) and (16) for $i=j$.

\section{Appendix B: Simple approximation for the escape probability}

In the Sobolev approximation, the escape probability for a line photon in the spherically symmetric accelerating outflow is given by (See e.g. Mihalas 1978, Sect. 14.2)

$\beta=\int_{0}^{1} \frac{1-\exp \left(-\chi_{\mathrm{l}} / Q(\mu)\right)}{\chi_{\mathrm{l}} / Q(\mu)} \mathrm{d} \mu$,

where

$Q(\mu)=\mu^{2} \partial V / \partial r+\left(1-\mu^{2}\right)(V / r)$,

$\chi_{\mathrm{l}}=\frac{\left(\pi e^{2} / m c\right) f}{\Delta \nu_{\mathrm{D}}} n$,

$V=v / v_{\text {th }}, v$ and $v_{\text {th }}$ are the outflow and scattering atoms thermal velocities respectively, we set $\Delta \nu_{\mathrm{D}}=\nu v_{\mathrm{th}} / c, \nu$ is the line frequency, $f$ is the oscillator strength, and $n$ is the number density of atoms on the lower level of the transition.
Here and in what follows we consider the fixed point of the envelope at the distance $r$ from the center and do not show explicitly the dependence of $\beta, V, n$, and other physical parameters of the envelope on $r$. Also, in expression for $\chi_{\mathrm{l}}$ we neglected the stimulated emission, which do not introduce a noticeable error when the formation of $\mathrm{H} \alpha$ in the $\mathrm{P}$ Cyg envelope is considered.

Our purpose is to find an approximation $\tilde{\beta}$ to $\beta$ that permits fast evaluation. By solving the equations of statistical equilibrium using the approximation instead of the exact definition, $\tilde{\beta}$ should retain principal mathematical properties of $\beta$ reflecting the underlying physics. Namely, we require that $\tilde{\beta}(0)=1$ and $\tilde{\beta}(n)$ should monotonically decrease to zero as $n \rightarrow \infty$.

The simplest function that obeys the above stated requirements is

$\tilde{\beta}=\frac{1}{1+n / n_{\text {as }}}$

where $n_{\text {as }}$ is a parameter. To choose the optimal value of $n_{\text {as }}$, we consider the behavior of $\beta(n)$ for large $n$. When $n \rightarrow \infty$, the numerator of the integrand in Eq. (B1) can be replaced by 1 and an asymptotic form of $\beta$ can be easily computed.

Comparison of that asymptotic form with Eq. (B2) shows that if we set

$n_{\mathrm{as}}=8 \pi \frac{g_{\mathrm{l}}}{g_{\mathrm{u}}} \frac{1}{\lambda^{3}} A_{\mathrm{ul}}\left(\frac{1}{3} \frac{\mathrm{d} v}{\mathrm{~d} r}+\frac{2}{3} \frac{v}{r}\right)$

then $\tilde{\beta} / \beta=1$ up to the terms of order $1 / n$ when $n \rightarrow \infty$. Here $g_{1}$ and $g_{\mathrm{u}}$ are statistical weights of the lower and upper levels of the transition respectively, $\lambda$ is the line wavelength, and $A_{\mathrm{ul}}$ is the spontaneous emission coefficient.

To study the precision of approximation (B2), we performed numerical computations for a wide range of values $(\mathrm{d} v / \mathrm{d} r) /(v / r)$ and $n / n_{\text {as }}$ (it can be easily shown that the error is uniquely determined by the values of these two parameters). Our results show that the relative error reaches its maximum of 0.23 for $\frac{\mathrm{d} v(r)}{\mathrm{d} r}=\frac{v(r)}{r}$, that is when $v(r) \propto r$, and $n_{2} / n_{\text {as }} \approx 1.8$.

The approximation found here is applicable if the escape probability is given by Eq. (B1), that is if the frequency change in scattering is described by complete frequency redistribution. Of course, this is useful only if the usual conditions of applicability of the Sobolev approximation are satisfied. The expressions (B2) and (B3) can be generalized in two ways. First, the error can be reduced if rational approximations of higher order are used instead of Eq. (B2). Second, a similar approximation can be obtained for a general three-dimensional flow as will be showed in a future study. 


\section{References}

Bertout C., 1984, ApJ 285, 269

Bourguine M.S., Chalabaev A.A., 1994, in: Pulsation, Rotation and Mass Loss in Early-Type Stars, IAU Symp. 162, Balona L.A., Henrichs H.F., Le Contel J.M. (eds.). Kluwer, Dordrecht, p. 502

Bracewell, 1978, The Fourier Transform and its applications. Mc Graw Hill, New York

Burgin M.S., Chalabaev A.A., 1992, in: ESA Colloquium on Targets for Space-based Interferometry, Beaulieu, October 1992, France, ESA SP-354, p. 185

Casatella A., Benvenuti P., Clavel J., Heck A., Penston M., Selvelli P.L., Beekmans F., Lamers H.J.G.L.M., Machetto F., Stickland D., 1979, A\&A 149, 223

Castor J.I., 1970, MNRAS 149, 111

Drew J.E., 1985, MNRAS 217, 867

Goodman J.W., 1985, Statistical Optics. Willey Interscience

Hamann W.-R., 1981, A\&A 93, 353

Harmanec P., Morand F., Bonneau D., et al., 1996, A\&A 312, 879

Humphreys R.M., Davidson K., 1994, PASP 106, 1025

Kendall M.G., Stuart A., 1967, Inference and relationship (2nd ed.). Charles Griffin \& Co. Ltd, London

Lamers H.J.G.L.M., de Groot M., Casatella A., 1983, A\&A 128,299

Lamers H.J.G.L.M., de Koervaar P., Casatella A., 1985, A\&A 149,29

Lamers H.J.G.L.M., Cerruti-Sola M., Perinotto M., 1987, ApJ 314,726

Langer N., Hamann W.-R., Lennon M., Najarro F., Pauldrach A.W.A., Puls J., 1994, A\&A 290, 819

von der Lühe O, Bonaccini D., Derie F., Koehler B., Léveque S., Manil E., Michel A., Verola M., 1997, The ESO Messenger, 87

McAlister H.A., Bagnuolo W.G., Ten Brummelaar T., Hartkopf W.I., Turner N.H., Shure M.A., Sturmann L., Turner N.H., Ridgway S.T., 1998, SPIE 3350, 847

Maeder A., 1997, in: Luminous Blue Variables: Massive Stars in transition, Nota A. \& Lamers H. (eds.), ASP Conf. Ser. 120,374

Mariotti J.-M., 1988, in: Difraction-Limited Imaging with Very
Large Telescopes, Alloin D.A., and Mariotti J.-M. (eds.), p. 3

Mourard D., Bosc I., Labeyrie A., Koechlin L., Saha S., 1989, Nat 342,520

Mourard D., Tallon-Bosc I., Rigal F., Vakili F., Bonneau D., Morand F., Stee Ph., 1994, A\&A 288, 675

Mihalas D., 1978, Stellar atmospheres (2nd ed.). Freeman, San Fransisco

Nota A., 1998, in: Variable and Non-spheric Stellar Winds in Luminous Hot Stars, Proc. of IAU Coll. 169, Heidelberg, 15-19 June 1998, Wolf B., Stahl O., Fullerton A.W. (eds.). Springer, p. 62

Pauldrach A.W.A., Puls J., 1990, A\&A 237, 409

Petrov R.G., Malbet F., Richichi A., Hofmann K.-H., 1998, The ESO Messenger 92, 11

Per̂ina J., 1972, Coherence of light. Van Notsrand Reinhold Co., London, Ch. 3

Roddier F., Léna P., 1984, J. Opt. 15, 4, 171

Quirrenbach A., Hummel C.A., Buscher D.F., Armstrong J.T., Mozurkewich D., Elias N.M., 1993, ApJ 416L, 25

Quirrenbach A., Buscher D.F., Mozurkewich D., Hummel C.A., Armstrong J.T., 1994, A\&A 283L, 13

Quirrenbach A., Bjorkman K.S., Bjorkman J.E., Hummel C.A., Buscher D.F., Armstrong J.T., Mozurkewich D., Elias N.M., Babler B.L., 1997, ApJ 479, 477

Scuderi S., Bonanno G., Spadaro D., Panagia N., Lamers H.J.G.L.M., de Koter A., 1994, ApJ 437, 465

Stee P., De Araujo F.X., Vakili F., Mourard D., Arnold L., Bonneau D., Morand F., Tallon-Bosc I., 1995, A\&A 300, 219

Taylor M., Nordsieck K.H., Schulte-Ladbeck R.E., Bjorkman K.S., 1991, AJ 102, 1197

Vakili F., Mourard D., Stee P., 1994, in: Pulsation, Rotation and Mass Loss in Early-Type Stars, IAU Symp. 162, Balona L.A., Henrichs H.F., Le Contel J.M. (eds.). Kluwer, Dordrecht, p. 435

Vakili F., Mourard D., Bonneau D., Morand F., Stee P., 1997, A\&A 323, 183

Wolf B., Stahl O., Fullerton A.W., 1998, Variable and Nonspheric Stellar Winds in Luminous Hot Stars, Proc. of IAU Coll. 169, Heidelberg, 15-19 June 1998. Springer 